\title{
Magnetic Field Extrapolations into the Corona: Success and Future Improvements
}

\author{
S. Régnier ${ }^{1}$

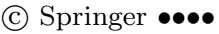

\begin{abstract}
The solar atmosphere being magnetic in nature, the understanding of the structure and evolution of the magnetic field in different regions of the solar atmosphere has been an important task over the past decades. This task has been made complicated by the difficulties to measure the magnetic field in the corona, while it is currently known with a good accuracy in the photosphere and/or chromosphere. Thus, to determine the coronal magnetic field, a mathematical method has been developed based on the observed magnetic field. This is the so-called magnetic field extrapolation technique. This technique relies on two crucial points: (i) the physical assumption leading to the system of differential equations to be solved, (ii) the choice and quality of the associated boundary conditions. In this review, I summarise the physical assumptions currently in use and the findings at different scales in the solar atmosphere. I concentrate the discussion on the extrapolation techniques applied to solar magnetic data and the comparison with observations in a broad range of wavelengths (from hard $\mathrm{X}$-rays to radio emission).
\end{abstract}

Keywords: Corona, Models - Corona, Structures - Magnetic Fields, Models Active Regions

\section{Introduction}

With the advent of photospheric magnetographs/magnetometers in the sixties, it has soon been realised that the coronal magnetic field can be derived by assuming an equilibrium state. The main forces excerted on the coronal plasma are the plasma pressure, the gravitational force, and the magnetic or Lorentz forces. This gives the magnetohydrostatic equilibrium:

$$
-\nabla p+\rho \mathbf{g}+\mathbf{j} \times \mathbf{B}=\mathbf{0}
$$

where $p$ and $\rho$ are the plasma pressure and density, $\mathbf{g}$ is the gravity, $\mathbf{j}$ is the electric current density, and $\mathbf{B}$ is the magnetic field. This equation is a partial

\footnotetext{
1 Jeremiah Horrocks Institute, University of Central Lancashire, Preston, Lancashire, PR1 2HE, United Kingdom email: SRegnier@uclan.ac.uk
} 
different equation, which can be solved by imposing a set of boundary conditions in a finite or semi-finite domain of computation. In addition, Maxwell's equation given the divergence-freeness of the magnetic field has also to be considered. The technique is the so-called magnetic field extrapolation/reconstruction (nowadays both words are used without distinction). Two problems have to be distinguished: (i) to numerically solve the system of equations, (ii) to incorporate solar data into the numerical codes. Both problems need a careful treatment as the convergence of a well-established numerical code does not imply the convergence of the same code with a specific dataset. In this review, I will address the magnetic field extrapolations performed using photospheric/chromospheric magnetograms as boundary conditions. This indeed assumes that the algorithms to extrapolate the magnetic field are behaving well with data.

The magnetograms used as boundary conditions are recorded by groundbased or space-born instruments. For this review, the pros and cons of different instruments are not taken into account and only the physics problems and results addressed in the cited papers are considered. The aim of this review is to show that the magnetic field extrapolation techniques have been used to tackle successfully a broad range of solar physics issues.

Recently, two reviews have been published with a different focus to this paper: (i) Wiegelmann and Sakurai (2012) focused their discussion on the nonlinear force-free field methods with a short section on the comparison with the structures of the solar corona, (ii) Mackay and Yeates (2012) describe the progress in the modelling of global photospheric and coronal magnetic fields which includes extrapolation techniques as well as flux transport models which are not discussed here.

The review is organised as follows. I first give a brief review of the different models used to reconstruct the solar atmospheric magnetic field (see Section 2). In Section 3, the important quantities that can be derived from a magnetic field configuration are listed. Thus, the results obtained from magnetic field extrapolations are discussed for the quiet Sun (see Section (4), and for active regions (see Section 5). To conclude (see Section 6), I discuss the issues which are still to be resolved, and the futher developments that I envision for the magnetic field extrapolation techniques.

\section{A Brief Review of Numerical Models}

Magnetic field extrapolation techniques aim at solving a system of differential equations with the appropriate set of boundary conditions. An extrapolation scheme is judged on the well-posedness of the problem, that is to say to find the right combination of boundary conditions to solve a particular physical problem. I will thus distinguish between (i) the goodness of the numerical scheme which is usually tested with analytical and/or semi-analytical solutions, and (ii) the goodness of the extrapolation which includes the effects of the boundary conditions as provided by observations. Most of the schemes described below are good numerical schemes; however, their behaviour when extrapolating solar data can be significantly different, or has not yet been tested. 


\subsection{Potential Fields}

The potential field is the solution of Laplace's equation:

$$
\nabla^{2} \mathbf{B}=0
$$

The solutions of this equation are well-known as harmonic functions in different geometries. The potential field is relatively easy to compute as it only requires the knowledge of the normal component of the magnetic field on the boundaries of the domain. For instance, to compute the potential field in a Cartesian box only the normal/vertical component is needed on the bottom boundary as often provided by line-of-sight magnetic field measurements, whilst closed/open boundary can be imposed on the side and top boundaries. The first potential field extrapolation techniques have been in the early 60 s with the development of photospheric magnetographs (Schmidt, 1964, Altschuler and Newkirk, 1969; Levine and Altschuler, 1974: Schatten, Wilcox, and Ness, 1969: Adams and Pneuman, 1976 Levine, Schulz, and Frazier, 1982||Hakamada, 1995||Rudenko, 2001). Several methods have been compared by Seehafer (1982) showing that the connectivity and geometry of field lines can be different fom one model to the other.

\subsection{Force-free Fields}

We usually distinguish between potential field and force-free field extrapolations even if the potential field is a particular case of force-free field, and thus has similar properties of existence and stability (Molodensky, 1974). The force-free fields assume implicitely that the solar atmospheric plasma is a low $\beta$ plasma.

\subsubsection{Linear Force-free Field}

The linear force-free (LFF) field is described by the following equation:

$$
\nabla \times \mathbf{B}=\alpha \mathbf{B}
$$

where $\alpha$ is a scalar called the force-free parameter. The boundary conditions are given by the normal component of the magnetic field on the boundaries of the computational box, to which a guess for the $\alpha$-value is added. The numerical methods to solve this linear problem include vertical integration, Green's functions, Fourier transforms, spherical harmonics, and boundary integrals in either Cartesian or spherical coordinates (Nakagawa and Raadu, 1972, Levine and Altschuler, 1974: Seehafer, 1975, Chiu and Hilton, 1977; Nakagawa, Wu, and Tandberg-Hanssen, 1978, Alissandrakis, 1981; Wu and Wang, 1984, 1985, Semel, 1988; Durrant, 1989, Gary, 1989, Yan, 1995, Abramenko and Yurchishin, 1996, Kusano and Nishikawa, 1996, Amari, Boulmezaoud, and Maday, 1998, Clegg, Bromage, and Browning, 1999, Clegg et al., 2000). Very few studies of the behaviour of LFF field algorithms have been performed (Li, Song, and Li, 2009).

As it is obvious from photospheric vector magnetic field observations that a single value of $\alpha$ cannot describe the coronal magnetic field (Leka and Skumanich, 1999 Leka, 1999), several methods have been attempted to derive the geometry of 
a single coronal loop using the $\alpha$-value that will best fit the observed loop (Wiegelmann and Neukirch, 2002, Carcedo et al., 2003). In other words, this corresponds to a piecewise LFF field for a discrete (usually small) number of loops.

\subsubsection{Nonlinear Force-free Field}

The nonlinear force-free (NLFF) field satisfies the following equation:

$$
\nabla \times \mathbf{B}=\alpha(\mathbf{r}) \mathbf{B}
$$

where $\alpha$ is the force-free function depending on the position $\mathbf{r}$. Taking the divergence of the above equation, we obtain that

$$
\text { B } \cdot \nabla \alpha=0
$$

implying that $\alpha$ is constant along a given magnetic field line. This fact is reinforced in the corona as the conduction along the magnetic field dominates (the conduction accross field lines is negligible). The NLFF methods have been summarised by Wiegelmann and Sakurai (2012). The main methods to extrapolate the magnetic field into the corona as a NLFF field are:

- Vertical integration: the method consists in propagating the boundary conditions into the corona from the bottom boundary, layer by layer ( $\mathrm{Wu}$ and Wang. 1985. Cuperman, Ofman, and Semel, 1989, 1990a, 1990b, Wu et al., 1990, Démoulin, Cuperman, and Semel, 1992, Song et al., 2006, 2007).

- MHD evolutionary techniques: based on the low plasma- $\beta$ MHD equations, an initial configuration including electric currents is relaxed to a nonlinear force-free state owing to resistivity, also know as stress-and-relax models (Yang, Sturrock, and Antiochos, 1986: Mikic, Barnes, and Schnack, 1988, Schnack et al., 1990 McClymont and Mikic, 1994|:Roumeliotis, 1996:|Valori, Kliem, and Keppens, 2005 Valori, Kliem, and Fuhrmann, 2007; Valori et al., 2010; Jiang et al., 2011).

- Optimization: the basic principle is to minimise a functional containing the force-free constraint as well as the solenoidal constraint to relax an initial configuration towards a nonlinear force-free state (Wheatland, Sturrock, and Roumeliotis, 2000: Wiegelmann, 2004, Wiegelmann et al., 2005, 2008, 2010, Wiegelmann, Inhester, and Sakurai, 2006, Inhester and Wiegelmann, 2006, Wiegelmann and Neukirch, 2006, Tadesse, Wiegelmann, and Inh 2009, Mysh'yakov and Rudenko, 2009, Wiegelmann and Inhester, 2010, Fuhrmann et al., 2011). In the recent years, the optimization scheme has been developed to include more constraints and thus to obtain an equilibrium closer to a force-free field.

- Grad-Rubin methods: the nonlinear force-free equation being a system of partial differential equations of mixed type, the method described by Grad and Rubin] (1958) consists in separating the elliptic part (force-free equation) and the hyperbolic part (gradient of $\alpha$ ) of the system, each system being then linear and easier to solve (Grad and Rubin, 1958, Sakurai, 1981, Amari et al., 1997; Amari, Boulmezaoud, and Mikic, 1999: Wheatland, 2004:Amari, Boulmezaoud, and Aly, 2006 Inhester and Wiegelmann, 2006; Wheatland, 2006; Wheatland and Régnier, 2009 
Malanushenko, Longcope, and McKenzie, 2009; Amari and Aly, 2010; Malanushenko et al., 2012).

The Grad-Rubin method requires as boundary conditions the vertical/radial component of the magnetic field on each boundary, as well as the distribution of $\alpha$ in a chosen polarity. This ensures that the extrapolation method is a mathematically well-posed problem in Hadamard sense.

- Boundary integral: the nonlinear force-free model can be describe as an exterior problem and a boundary integral equation written in which the magnetic field component within a volume can be determined by the magnetic field components on the boundary. The formulation of the boundary integrals can be found by Courant and Hilbert (1963) and recently applied to solar cases (D. Wang, Wei, and Yan, 1995; Yan and Sakurai, 1997, 2000; Li, Yan, and Song, 2004, He and Wang, 2006; Yan and Li, 2006, He and Wang, 2008).

- Force-free electrodynamics: the theory of force-free electrodynamics is applied to the modelling of coronal magnetic fields which has been applied successfully to pulsar magnetospheres (Contopoulos, Kalapotharakos, and Georgoulis, 2011 Contopoulos, 2013).

With the development of different numerical techniques, testing and comparing different algorithms has been proven to be needed. The main efforts have been done since 2004 by the international group on nonlinear force-free modelling (Schrijver et al., 2006, 2008, Metcalf et al., 2008, De Rosa et al., 2009). One aim of this series of papers has been to show that modellers need to be careful when injecting the boundary conditions into the numerical codes: the boundary conditions have to be consistent with the assumption of the model and the set-up of the numerical code. For instance, in Schrijver et al. (2008), it has been shown that by selecting carefully the photospheric magnetic field, the coronal magnetic field structure in the core of the reconstructed active region was similar for all NLFF methods. Several other papers have addressed quantitative differences between several algorithms (Inhester and Wiegelmann, 2006; Barnes, Leka, and Wheatland, 2006; Rudenko and Myshyakov, 2009; Mysh'yakov and Rudenko, 2009: Liu et al., 2011).

\subsection{Non-force-free Fields}

The next step after the force-free field extrapolation is to solve the magnetohydrostatic (MHS) equilibrium equation, which includes the plasma pressure force and the gravitational force in addition to the magnetic forces. An algorithm has been developed by Grad and Rubin (1958) as a well-posed problem. This algorithm has been recently implemented by Gilchrist and Wheatland (2013) neglecting the gravity (see also Boulbe, Zamène Boulmezaoud, and Amari 2009). The optimization scheme has also been adapted to solve the magnetohydrostatic equation (Wiegelmann and Neukirch, 2006: Wiegelmann et al., 2007). The mod-I els usually require to define a realistic atmosphere from the photosphere to the corona. As a first order assumption (neglecting the feedback between the magnetic field and plasma properties), Régnier, Priest, and Hood (2008) derived the global properties of Alfvén speed, plasma $\beta$-values and reconnection rate in 
the corona by assuming a nonlinear force-free equilibrium for the magnetic field and a hydrostatic equilibrium for the plasma.

A minimum dissipation rate method has been adapted to include photospheric measurements ( $\mathrm{Hu}$ and Dasgupta, 2006, 2008: Bhattacharyya et al., 2007: Hu et al., 2008 , 2010). The method describes a general or non-force-free magnetic field by a superimposition of one potential field and two linear force-free fields.

Gary and Alexander (1999) have developed a non-force-free model, the socalled stretched magnetic field, which combined photospheric magnetograms and the structures of coronal loops.

\subsection{Miscellaneous}

I will also mention several other analytical/numerical methods that have been used to access the structure of the three-dimensional coronal magnetic field, but which cannot be classified as extrapolation techniques as such.

This first method to have produced significant results is the point-charge method. A magnetogram is decomposed in a discrete number of polarities, which are reduced to points associated with the magnetic flux of the polarity (zero magnetic field outside the point charges). The magnetic field is thus given by an analytical solution. For instance, the method has been developed and used for theorising the topology of magnetic field in different geometries (Titov, Priest, and Demoulin, 1993; Bungey, Titov, and Priest, 1996; Brown and Priest, 1999, Longcope and Klapper, 2002; Beveridge, Priest, and Brown, 2002, 2004, Barnes, Longcope, and Leka, 2005, Maclean et al., 2006, Maclean, Beveridge, and Priest, 2006). The point-charge method has also been used to fit the observed 3D coronal loops following a nonlinear force-free assumption (Aschwanden, 2012a, 2012b, 2013, Aschwanden and Malanushenko, 2012). Another successful model to determine the properties of the coronal magnetic field is the flux rope insertion model developed by van Ballegooijen (2004). Starting from an equilibrium state (often a potential field), a flux rope or twisted flux tube is inserted within the magnetic configuration by modifying the boundary conditions and by matching the observed X-ray sigmoid or $\mathrm{H} \alpha$ filament (see e.g., van Ballegooijen et al., 2007).

\subsection{Boundary Conditions}

In order to solve a system of differential equations, it is important to define the correct boundary conditions that will lead to a mathematically well-posed problem (or not) in the Hadamard sense.

For the potential field, only the normal component of the magnetic field on the boundaries of the computational box is needed.

For the linear force-free field, the normal component is required, to which a guess for the value of $\alpha$ in the computational volume will be added.

For the nonlinear force-free field, the normal component of the magnetic field as well as the distribution of $\alpha$ has to be imposed. According to Grad and Rubin (1958), imposing the distribution of $\alpha$ in one chosen polarity as the boundary condition will lead to a mathematically well-posed problem. It is important to 
notice here that in order to compute the distribution of $\alpha$ from vector magnetograms, the following formula is used (in Cartesian coordinates with $(x, y)$ on the photospheric surface):

$$
\alpha=\frac{1}{B_{z}}\left(\frac{\partial B_{y}}{\partial x}-\frac{\partial B_{x}}{\partial y}\right) .
$$

This equation is considered owing to the measurement of the magnetic field vector on the $x y$-plane, implying that no derivatives with respect to $z$ can be computed. A further discussion on the implication of this formula will be done in Section 6.2

For the minimum dissipation model, the vector magnetic field components on one or two different atmospheric layers are needed.

For the magnetostatic model, the same boundary conditions as for the nonlinear force-free model are used in addition to prescribing the plasma pressure on the photospheric level in one chosen polarity. If the gravitational force is considered, then an initial atmosphere model will be needed.

\section{Physical Quantities}

Below, I list the important quantities that can be derived from the magnetic field extrapolations and which will lead to an in-depth physical interpretation of the magnetic field structure and coronal phenomena as described in Sections 4 and 5 .

\subsection{Magnetic Energy}

Magnetic Energy The magnetic energy computed in a volume $V$ is given by

$$
E_{\mathrm{m}}=\int_{V} \frac{B^{2}}{2 \mu_{0}} d V .
$$

The reconstruction models provide the three components of the magnetic field at discrete locations in the computational volume. The magnetic energy can then be easily computed by discretizing Equation (7). The magnetic energy for the potential field is a minimum (lower bound) of magnetic energy for a force-free field computed with the same boundary conditions, i.e., the same vertical/normal magnetic component on all boundaries. The LFF magnetic energy is a minimum of magnetic energy for the NLFF field if the magnetic helicity is conserved (Woltjer, 1958, Taylor, 1974).

Free Magnetic Energy The free magnetic energy is the difference in magnetic energy for a magnetic field model and for a reference field:

$$
\Delta E_{\mathrm{m}}=E_{\mathrm{mod}}-E_{\text {ref }}
$$

The reference field is often chosen to be the potential magnetic field as it is the minimum energy state (Aly, 1984). The free magnetic energy is a measure of the magnetic energy that can be stored in the magnetic configuration or released during an eruptive or reconnection event. 
Magnetic Energy Density The magnetic energy density is the term $\frac{B^{2}}{2 \mu_{0}}$ taken at one particular location within the volume $V$. The energy density can also be seen as the magnetic energy in a small volume $\delta V$ (e.g., a single pixel). As such, the magnetic energy density provides the distribution of magnetic energy in a small volume. However, the free magnetic energy density is of no use/meaning as the connectivity of magnetic field lines between the magnetic field and a reference field can be drastically different.

The Aly-Sturrock Conjecture Both Aly (1989) and Sturrock (1991) showed that there exists an upper bound of the magnetic energy of force-free fields when the field is totally open (unipolar field). The condition of application of this conjecture is when the magnetic field within the volume decays rapidly towards the boundaries, or when the magnetic flux through the boundaries other than the bottom boundary becomes negligible. This condition is easily satisfied when considering the half space above the photosphere, or when the boundaries are far enough from the magnetic field sources. This condition is not satisfied when magnetic flux is present near the edges of the boundaries.

Time Evolution One of the most interesting physical issues about magnetic extrapolation has been to know if the reconstructed magnetic field can describe the time evolution of solar regions. In the non-eruptive solar corona, the time of evolution is mostly given by the Alfvén transit time along individual magnetic field lines or coronal loops for active regions, and by the comparison of Alfvén transit time and granular motion for the quiet Sun. For active regions, the Alfvén transit time of a typical loop of length $200 \mathrm{Mm}$ is about 10-15 min; therefore if there is no major injection of magnetic energy or magnetic helicity, the evolution of active regions can be studied by a time series of equilibria.

\subsection{Magnetic Helicity}

General Definition The concept of magnetic helicity has been largely used in plasma physics to describe the complexity of the magnetic field, including the twist and shear in magnetic field lines. Maxwell's equation,

$$
\nabla \cdot \mathbf{B}=0
$$

implies that the magnetic field is solenoidal and can be described by a vector potential $\mathbf{A}$ such that $\mathbf{B}=\nabla \times \mathbf{A}$. The definition of $\mathbf{A}$ is not unique and depends on a gauge condition. From this definition, the magnetic helicity in a volume $V$ is defined as

$$
H_{\mathrm{m}}=\int_{V} \mathbf{A} \cdot \mathbf{B} d V=\int_{V} \mathbf{A} \cdot \nabla \times \mathbf{A} d V .
$$

The main concepts about magnetic helicity have been summarised by Berger (1999a). 
Relative Magnetic Helicity As the definition of Equation (10) is not unique, a gauge invariant definition has been developed by Berger and Field (1984)

$$
\Delta H_{\mathrm{m}}=\int_{V}\left(\mathbf{A}-\mathbf{A}_{\mathrm{ref}}\right) \cdot\left(\mathbf{B}+\mathbf{B}_{\mathrm{ref}}\right) d V
$$

and also by Finn and Antonsen (1985)

$$
\Delta H_{\mathrm{m}}=\int_{V}\left(\mathbf{A}+\mathbf{A}_{\mathrm{ref}}\right) \cdot\left(\mathbf{B}-\mathbf{B}_{\mathrm{ref}}\right) d V .
$$

The definition of Berger and Field (1984) originally contained a surface term, which tends to zero when considering the half space above the photosphere (or boundaries far away from the strong magnetic field regions) or vanishes for a finite volume when the following boundary conditions are imposed: the magnetic field normal to the surface of the finite volume is the same for both magnetic fields, the divergence of the reference vector potential vanishes, and the reference vector potential is perpendicular to the surface of the finite volume. In those two cases, the Finn and Antonsen (1985) and Berger and Field (1984) formulae are equivalent.

Magnetic Helicity Conservation In ideal MHD, the magnetic helicity is invariant during the evolution of any closed flux system (Woltjer 1958). Taylor (1974) applied this to laboratory experiments and hypothesized that, for a weak but finite resistivity, the total magnetic helicity of the flux system is invariant during the relaxation process. Taylor's theory has often been invoked to assume that the magnetic helicity in a solar atmospheric region is conserved. This latter statement is valid only if the solar region under consideration is a closed flux system. For instance, active regions in which flux emergence is taking place or a CME has originated cannot satisfy helicity conservation. The redistribution of magnetic helicity at large scale in the solar atmosphere or in the heliosphere is a basic consequence of the magnetic cycle and the sustainability of the dynamo action.

Woltjer (1958) has shown that the minimum energy of a NLFF field is a LFF field when the magnetic helicity is conserved (i.e., for a closed system). This implies that the difference between the magnetic energy of the NLFF field and LFF field is a better estimate of the free magnetic energy (see Section 3.1).

Self and Mutual Helicity The magnetic helicity of a magnetic configuration can be decomposed into several components: self and mutual helicity (Berger, 1999b), or twist and writhe (Berger and Prior, 2006). As an example, for a single twisted flux tube embedded a uniform external magnetic field, the self helicity is assumed to correspond to the twist within the flux tube, while the mutual helicity corresponds to the crossing between the external field and the twisted flux tube. The definitions given by Berger (1999b) have been studied in the frame of force-free extrapolation of active regions by Régnier, Amari, and Canfield|| (2005): the self helicity is related to the twist of the flux bundles within the active region, while the mutual helicity contains a contribution from the crossing of field 
lines and the large-scale twist of the active region (large compared to the size of the computational box). Longcope and Malanushenko (2008) have defined a formula for the self helicity which describes only the twist of the field lines.

Twist in Force-free Fields The magnetic helicity is a measure of the topology of a magnetic field configuration, and also includes a measure of the shear and twist of magnetic field lines. In the solar physics literature, the force-free parameter/function, $\alpha$, is often called the twist. This is a misnomer as $\alpha$ is strictly equivalent to the twist in a thin flux tube approximation, which does not always apply to the magnetic field extrapolated in solar regions. Especially in a non-idealised configuration, the twist and the $\alpha$-value can have opposite sign (Régnier and Amari, 2004, Prior and Berger, 2012).

Time Evolution As for the magnetic energy, the evolution of magnetic helicity injection and redistribution can be studied by a time series of equilibria. The time scale of the change in magnetic helicity is shorter than the time scale for the magnetic energy. A general equation for the rate of change of magnetic helicity has been derived by Heyvaerts and Priest (1984) for a non-ideal plasma.

Other Helicities Magnetic helicity is the integral over a given volume of the vector potential A and its curl, B. This definition can be extended to other quantities such as the electric current density or the vorticity. The current helicity is

$$
H_{\mathrm{C}}=\int_{V} \mathbf{j} \cdot \mathbf{B} d V
$$

with $\nabla \times \mathbf{B}=\mu_{0} \mathbf{j}$, and the hydrodynamical helicity by

$$
H=\int_{V} \mathbf{v} \cdot \omega d V
$$

where $\mathbf{v}$ is the flow field and $\omega=\nabla \times \mathbf{v}$ is the associated vorticity.

From vector magnetograms, the current helicity on the surface has been estimated either by assuming that the transverse components of the magnetic field and/or the electric current density are negligible, or by assuming a LFF field (e.g., Abramenko, Wang, and Yurchishin 1996). For the latter assumption, the current helicity density is $h_{\mathrm{c}}=\alpha B^{2}$. For a volume $V$, the current helicity associated with a LFF field is

$$
H_{\mathrm{C}}=\int_{V} \alpha B^{2} d V=2 \mu_{0} \alpha E_{m}
$$

where $E_{\mathrm{m}}$ is the magnetic energy.

\subsection{Magnetic Topology}

As it is beyond the scope of this review to give a complete description of all topological studies, I will just mentioned that the concept and development of magnetic topology applied to coronal structures have been reviewed in Longcope 
(2005). The transfer of magnetic energy and magnetic helicity through topological elements is the consequence of magnetic reconnection, and thus eruptive events. In particular, the main ingredients are null points where the magnetic field vanishes, separators, separatrices, quasi-separatrices, and hyperbolic flux tubes.

The magnetic topology of the potential field is assumed to be just slightly modified compared to other magnetic field models (Hudson and Wheatland, 1999 Brown and Priest, 2000). It has recently been shown by Régnier (2012) that the location and properties of null points existing in a simple configuration for potential, LFF, and NLFF fields are similar when the spectral radius (maximum in the absolute value of the eigenvalues associated with the null point) is large, meaning that the magnetic null point is embedded in a strong field region or surrounded by strong electric currents (i.e., large magnetic field gradients).

Null points have been extensively used as a proxy to the complexity of the magnetic field and the possible existence of reconnection events in a diffusion region encompassing the null points. This can be extended to the study of current sheets. The diffusion regions including a null point or degenerated from a null point are supposed to be the more efficient in releasing magnetic energy and to convert this energy into kinetic and thermal energies. Priest, Longcope, and Heyvaerts| (2005) have pointed out that the magnetic energy storage induced by slow photospheric motions is much more efficient along separators than at separatrices.

Another quantity that has been commonly used is the $Q$-factor or squashing degree (Titov, 2007). The $Q$-factor is a measure of the change of connectivity between neighbouring field lines and, as such, indicates the location where the magnetic energy could be dissipated or released. Except few quantitative studies (e.g., Longcope et al. 2010), only qualitative comparisons with observations have been performed.

\section{Quiet Sun}

\subsection{Validity of the Extrapolation Methods}

The complex physical behaviour of the photosphere and chromosphere makes the existence of electric currents perpendicular to the magnetic field possible, especially Hall currents described by the Hall parameter (ratio of the electron gyrofrequency to the electron-ion/neutral collision frequency), and the Pedersen currents (implied by convective electric field). These perpendicular currents will dissipate rapidly with altitude in the solar atmosphere (Gold and Hoyle, 1960; Goodman, 2000), leading to a force-free corona (only with parallel currents). The existence of such electric currents are crucial for the validity of the extrapolation methods in the quiet Sun. It is less important for active regions owing to the characteristic time scale of evolution.

Extrapolation methods such as potential fields have been applied to the quiet Sun without a real physical justification, but mostly due to the lack of observations/boundary conditions which will allow a better physical description of the system. The quiet-Sun magnetic field extrapolations can be regarded as a 
preliminary step towards the small scales of the solar atmosphere. It is also worth mentioning that the measurement of the magnetic field vector in the quiet-Sun regions is still challenging.

\subsection{The Magnetic Carpet}

The nature and structure of the magnetic field in quiet-Sun regions have been investigated with the means of magnetic field extrapolation. As currently line-ofsight magnetic field is the only reliable magnetic field component measured with high accuracy, the potential field model has been used for the quiet Sun. Note that the interest about the quiet-Sun evolution has recently been rejuvenated by nonlinear force-free modelling in a theoretical context (Meyer et al., 2011; Meyer, Mackay, and van Ballegooijen, 2012).

In 1999, Woodard and Chae have used a potential field model to show that the orientation of the $\mathrm{H} \alpha$ fibrils did not match the potential field lines, and thus concluding that the quiet-Sun magnetic field contains a non-potential component (see also Jing et al., 2011). H $\alpha$ fibril orientation has also been compared with linear force-free models by Nakagawa, Raadu, and Harvey (1973) finding that there is a value of $\alpha$ giving a good match between the magnetic field and the fibril direction; however the height of the structures was the largest discrepancy between the observations and the model. This fact has been justified by the existence of perpendicular electric currents in the photosphere and chromosphere such as Pedersen and Hall currents that will be dispersed with height to vanish in the corona. The 3D modelling of the magnetic carpet has been performed based on the point charge technique (Schrijver and Title, 2002): the assumption is justified in the quiet Sun as small-scale magnetic features can be isolated and tracked in time (DeForest et al., 2007, Lamb et al., 2008, 2010). Schrijver and Title (2002) showed that EUV brightenings can be correlated with the change of connectivity in the small-scale magnetic field. Building on this work, Régnier, Parnell, and Haynes (2008) performed a potential field extrapolation (with continuous magnetic field) using a high-resolution Hinode/SOT magnetogram to describe the complexity of the quiet-Sun magnetic field. The authors showed that the complexity of the quiet Sun is located in the photosphere and chromosphere at altitude less than $2.5 \mathrm{Mm}$ above the surface. In this modelling, the magnetic field in the quiet Sun has been decomposed into two components: (i) closed field lines, which characterise the complexity of the magnetic field (below several hundreds of kilometer according to Régnier, Parnell, and Haynes 2008) and are linked to granule's boundary and part of supergranular field, (ii) open field lines ("open" meaning leaving the box of computation), which is often considered as the source of the solar wind. The amount of open flux has been measured at different height showing that a small amount of the photospheric magnetic flux is open above few megameters. The imbalance of magnetic flux increasing with latitude (towards the poles), the open magnetic flux increases also with latitude. Jin and Wang (2011) showed that the open magnetic field does not always originate from strong polarities ( $\mathrm{kG}$ field) in the poles using vector magnetic field measurements from Hinode/SOT. It is worth noticing that the quiet-Sun magnetic field, and in particular the funnel 
structure, has been theoretically investigated using potential field extrapolation by Aiouaz and Rast (2006).

\subsection{Eruptive Events}

There are several eruptive events in the quiet Sun or small-scale events which require high-resolution field extrapolations: blinkers, jets, spicules, Ellerman bombs, or mini-CMEs. There is few studies tackling the time evolution of the events themselves, but mostly analysing the structures associated with these events.

Bright points (often observed in EUV or X-rays) have been studied in details in term of their magnetic field structure and time evolution. Based on the point charge technique, Alexander, Del Zanna, and Maclean (2011) have shown that the observed structure of the magnetic field (or some field lines used as a proxy to the magnetic field) is close to a potential field state.

With the development of high-resolution instrumentation, the magnetic field at a granular scale becomes available, and thus also the study of photospheric and chromospheric phenomena in the quiet Sun or active regions. Based on the Flare Genesis Experiment observations (Bernasconi, Rust, and Eaton, 2001), Pariat et al.! (2004) have studied the possibility of undulatory emergence in a small-scale field (a coherent flux tube emerging at different locations depending on the convection pattern) based on a LFF field extrapolation. The authors have noticed that the emergence locations are associated with Ellerman bombs. Guglielmino et al. (2010) studied reconnection events in a small-scale magnetic field within an active region during the emergence of magnetic flux. Using a LFF field extrapolation, the authors have derived the complexity associated with these events: the small-scale field exhibits a complex topology with a fan-spine structure.

From a potential extrapolation, He et al. (2010a) have shown that a jet occurring in a polar coronal hole is guided by the open magnetic field, and thus can be a source for the fast solar wind.

\section{Active Regions}

Active regions and their related structures and phenomena have been extensively studied with the advent of line-of-sight magnetographs and vector magnetographs measuring strong fields (active region fields) with high accuracy.

\subsection{Structure of the Magnetic Field}

As magnetic field extrapolations are based on an equilibrium, the first step is to study the static structure of the magnetic field above an active region.

General properties as follows have been derived:

- The magnetic field decays with height, but not following a bipolar magnetic field relation (Régnier, Priest, and Hood, 2008). The magnetic field can decay by several orders of magnitude with height. 
- Stating the obvious, individual active regions have different structures in terms of shear and twist, which depends on their history (Régnier and Priest, 2007a, 2007b).

- Active regions have a complex distribution of electric current density generated by photospheric motions and by emergence from below the photosphere (Leka, 1996).

- In a statistical sense, the magnetic field lines are longer and higher in altitude when electric currents are present (Régnier and Priest, 2007a).

- The magnetic energy is stored mostly at the bottom of the corona (including the photosphere and chromosphere), and can also be stored in twisted flux bundles in the low corona (Régnier and Priest, 2007a).

- Active regions can be decomposed into two parts: the core, and the edge. The core is dominated by strong electric current density and thus has sheared and twisted magnetic field bundles, while the edge of the active region is less influenced by electric currents and exhibits more potential field lines (De Rosa et al., 2009).

In terms of magnetic energy, active regions have been founded to have a total magnetic energy between $10^{31}$ erg to $10^{34}$ erg mostly depending on the total unsigned photospheric flux and the electric currents. The magnetic helicity has a characteristic value of $10^{42} \mathrm{G}^{2} \mathrm{~cm}^{4}$ (Régnier and Canfield, 2006).

Lots of studies have involved a comparison of the active region structures obtained using different assumptions. Hudson and Wheatland (1999) and Régnier (2012) showed that, for a simulated magnetic configuration, the magnetic topology does not change much between different force-free models, even if the geometry and the magnetic energy are significantly modified.

\subsection{Filament, Sigmoid, and Twisted Flux Bundles}

One of the main success of LFF, NLFF, or MHS extrapolation methods has been to reconstruct twisted flux tubes that unambiguously exist in the corona.

Filaments/prominences are thought to be a coherent collection of twisted magnetic field lines forming a twisted flux tube/bundle. The force-free field extrapolation has been used to show that there indeed are. Using a NLFF model, Yan et al. (2001a) described an active-region filament as a magnetic rope or twisted flux bundle with three turns. This is a highly twisted flux tube, which has never been reproduced in magnetic field extrapolation to date. In order to store plasma/mass, the most plausible structures are magnetic dips in which magnetic curvature acts against gravity to sustain the plasma above the surface. Using a LFF approximation, Aulanier and Démoulin (1998) have identified a filament by finding the magnetic dips in the 3D magnetic field configuration (Aulanier et al., 1998; Aulanier et al., 1999). Based on the same model, Dudík et al. (2008) showed the complexity of a filament as it can be fragmented, and not just a single coherent structure, by parasitic polarities constituting the legs or barbs of the filament. Using a NLFF method, Régnier and Amari (2004) identified a filament as a dipped twisted flux bundle with a small number of turn (see also Canou et al., 2009, Canou and Amari, 2010). Guo et al. (2010a) 
showed that part of a filament was identified to a twisted flux bundle, while the rest of the filament was correlated with magnetic dips along sheared untwisted field lines.

Sigmoids are expected to play an important role in solar eruptions (Canfield, Hudson, and McKenzie, 1999 They are considered as twisted flux bundles. Régnier, Amari, and Kersalé (2002) reproduced a sigmoid as a highly twisted flux tube without magnetic dips due to the nonuniform distribution of the twist along the structure. Other studies have been performed to understand the nature and evolution of X-ray sigmoid as twisted flux tubes (Savcheva, van Ballegooijen, and DeLuca, 2012: Savcheva et al., 2012 Inoue et al., 2012).

\subsection{Comparison with Observations}

The most common way of comparing observations and magnetic field extrapolation is qualitative: overlaying or putting side-by-side images and magnetic configurations. Some quantitative methods have been developed and are mentioned in the following paragraphs.

Optical Wavelengths The main feature observed in the visible wavelengths is a filament mostly in the $\mathrm{H} \alpha$ line. The findings using field extrapolations are summarised in the previous section. Structures like fibrils are not yet extrapolated due to their small height in the chromosphere; however their direction has been used to constrain the orientation of the transverse magnetic field in vector magnetograms (Wiegelmann et al., 2008). During flares, the chromospheric brightenings (localised patches or ribbons) have been identified as the footpoints of magnetic field lines linked to the flare process such as reconnection events or post-flare loop growth. Masson et al. (2009) used a potential field extrapolation to show the correlation between magnetic field lines involved in the flare process and chromospheric ribbons.

$\boldsymbol{E} \boldsymbol{U} \boldsymbol{V}-\boldsymbol{U} \boldsymbol{V}$ Using imagers, the EUV loops have been studied mostly at $171 \AA$ and 193/195 $\AA$ due to the characteristics of EUV instruments (e.g., SOHO/EIT, TRACE, STEREO/SECCHI/EUVI, SDO/AIA). A large number of studies has been performed to show the consistency of magnetic field extrapolations with observed EUV loops (Pallavicini, Sakurai, and Vaiana, 1981; Aschwanden, 2005; Zhang et al., 2007; Kwon and Chae, 2008: Aschwanden et al., 2008; Inhester, Feng, and Wiegelmann, 2008 Winebarger, Warren, and Falconer, 2008: Sandman et al., 2009: Conlon and Gallagher, 2010 Aschwanden and Sandman, 2010; Syntelis et al., 2012). The discrepancy between potential field lines and EUV loops has been used to show the existence of nonpotentiality in active regions, i.e., the existence of shear and twisted magnetic fields able to store magnetic energy and to trigger eruptions. Based on observations from (imaging) spectrometers, the extrapolation methods are compared to flows/Dopplershifts in active regions (Baker et al., 2019; Boutry et al., 2012).

Soft $\boldsymbol{X}$-rays Two main solar features observed in soft X-rays have been matched to magnetic flux bundles or magnetic field lines obtained from extrapolation: sigmoids (see Section 5.2) and X-ray bright points. The latter have been extensively studied since Golub et al. (1974). Adding magnetic extrapolations, the 
X-ray bright points have been studied in more detail (Mandrini et al., 1996; Brooks and Warren, 2008).

Hard $\boldsymbol{X}$-ray The hard X-ray sources are often observed during flares and reconnection events. The existence of sources at the footpoints of eruptive loops is well established, as well as the emission at the flare loop-top assumed to be located below the current sheet where the magnetic reconnection occur. The magnetic field extrapolations have helped to better understand the link between the geometry of loops, the topology of the magnetic field, and the sources of hard Xray (Takakura et al., 1983: Sakurai, 1985: Yan and Huang, 2003: Xu et al., 2010: Aurass et al., 2010; Liu et al., 2010: Guo et al., 2012).

Radio The radio emission can be observed during eruptive events such as flares, CMEs or filament eruptions, and it has been often suggested that the radio emission in the high corona occurs at the interface between closed and open magnetic field regions (Klassen et al., 1999; Bentley et al., 2000; Paesold et al., 2001; Aurass et al., 2003, 2005, 2011; Grechnev et al., 2006; Arzner and Vlahos, 2006; Yan et al., 2006, Hofmann and Ruždjak, 2007, Wen, Wang, and Zhang, 2007, Nitta and De Rosa, 2008, Klein et al., 2008, Chen et al., 2011;, Tun, Gary, and Georgoulis 2011, Del Zanna et al., 2011; Iwai et al., 2012; Hao et al., 2012). The gyroemission is also used to determine the strength of the magnetic field in the corona (Pallavicini, Sakurai, and Vaiana, 1981; Schmahl et al., 1982, Hildebrandt, Seehafer, and Krueger 1984, Brosius et al., 1997, 2002, Lee et al., 1998, 1999, Grebinskij et al., 2000, Ryabov et al., 2005, Bogod, Stupishin, and Yasnov, 2012). The comparison of those measurements and the magnetic field strength derived from extrapolation has yet not be proven to be satisfactory: the height of the radio sources does not always correlate.

Infrared Despite the development of solar observations in the infrared wavelengths, there is, to my knowledge, no study comparing the magnetic field derived from those observations (including the near-IR HeI triplet and Stokes parameter measurements in prominences by Paletou et al. (2001)) comparing the extrapolated magnetic field.

\subsection{Time Evolution}

The characteristic Alfvén transit time of an active region loop is of 10-15 min. The time evolution of an active region can thus be studied by a series of equilibria if the photospheric footpoint motions are ideal MHD motions, which do not add any topological constraints (Antiochos, 1987). The most complete study of the evolution of an active region has been performed by Sun et al. (2012) using a high-cadence, high-resolution time series of vector magnetograms from SDO/HMI. The authors studied the time evolution before and after a series of flares showing that the flare changes (slightly) the magnetic energy; the geometry of the magnetic field lines is also modified making the magnetic field more confined in low corona. For C-class flares, Régnier and Canfield (2006) have performed a study of the evolution of the magnetic energy and magnetic 
helicity. The authors showed that the start of the significant changes occur at about 20 min before the peak of the flare. Thalmann and Wiegelmann (2008) showed that the magnetic energy build-up in an active region can be observed several days prior to the eruption.

\subsection{Physics of Flares and CMEs}

One of the main early study of a flare using an extrapolation method corresponds to the Bastille Day flare (Aulanier et al., 2000). Using a LFF approximation, the authors showed that the flaring site was related to the existence of a null point located in the corona.

Another aspect of the physics of eruptions is the amount of magnetic energy that can be released. From a single snapshot (one extrapolation at a given time), the amount of free energy is estimated using the potential field as a minimum (lower bound) of magnetic energy. The magnetic energy of a LFF field with the same magnetic helicity can also be considered as a minimum of magnetic energy. Régnier and Priest (2007b) have demonstrated that the amount of free magnetic energy is larger than the energy of the flare associated with the active regions studied (only four active regions with very different structure have been studied). Numerous studies of flares and CMEs have been combined with magnetic field extrapolations to support the model of eruption (Schmidt, 1964; Zirin and Tanaka, 1973, Tanaka and Nakagawa, 1973; Rust, Nakagawa, and Neupert, 1975; Tanaka, 1978, Seehafer and Staude, 1979; Ma and Ai, 1979; Yang and Zhang, 1980; Su, 1980, 1982, Seehafer, 1985, Lin et al., 1985, Yang, Hong, and Ding, 1988; Lin, 1990, Linke, Ioshpa, and Selivanov, 1990; Klimchuk and Sturrock, 1992: Démoulin et al. 1994, McClymont and Mikic, 1994, Yan and Wang, 1995, Mandrini et al., 1995, Jiao, McClymont, and Mikic, 1997, Choudhary and Gary, 1999: Delannée and Aulanier, 1999, Yurchyshyn et al., 2000; Y. M. Wang, 2000; Yan et al., 2001a, 2001b; T. Wang et al. 2002, Yan and Huang, 2003, Moon et al., 2004, Fragos, Rantsiou, and Vlahos, 2004, Gary and Moore, 2004, Berlicki et al., 2004, Del Zanna et al., 2006; Li et al., 2007, Nitta and De Rosa, 2008, Jing et al., 2008, 2009, 2010, 2012, H. Wang et al. 2008: Martínez-Oliveros, Moradi, and Donea, 2008, Thalmann, Wiegelmann, and Raouafi, 2008, Guo et al., 2008, Zuccarello et al., 2009, Su et al., 2009a, 2009b; des Jardins et al., 2009 Chandra et al., 2009, He et al., 2010b; Xu et al., 2010, Cheng et al., 2010, Park et al., 2010; Liu et al., 2010, Guo et al., 2010b, Cheng et al., 2011; Inoue et al., 2011, Gilchrist, Wheatland, and Leka, 2012; Shen, Liu, and Su, 2012, Vincent, Charbonneau, and Dubé 2012, R. Wang et al., 2012; Georgoulis, Tziotziou, and Raouafi, 2012: Tadesse et al., 2012).

\section{Improvements and Challenges}

\subsection{Extrapolation Techniques}

Potential and LFF extrapolations are now mature techniques that are commonly used, and their physical meaning and the understanding of physical process are well established: no or limited amount of twist available in these models, and 
minimum energy state, for instance. However, the NLFF model is still in constant evolution/improvement. Since more than 20 years now, the NLFF assumption has been implemented in the solar community to study a large number of topics with a relatively good success. The next stages of development are:

- In Cartesian geometry, developing a reliable magnetohydrostatic equilibrium code. This corresponds to a step forward compared to the force-free field as far as the corona only is concerned. Including the thermodynamic properties of the photosphere and/or chromosphere is a challenge.

- In spherical geometry, improving the spatial resolution of the PFSS model is a first step, especially multigrids or nonuniform grids can be used. The PFSS model also currently lacks information at the poles: the improvement of the spatial resolution will need to be combined with a more accurate measure of the radial magnetic field at the poles. This will modify the dipole component of the magnetic field, which is needed to study the dynamo action.

- In spherical geometry, despite the efforts reported in previous the sections to move from the PFSS model to a NLFF assumption, the use of the NLFF model could become a standard in the community with a special care taken to the transition between the coronal magnetic field and the solar wind magnetic field. As for the NLFF field in Cartesian coordinates, a community effort should be envisioned.

- Defining contraints obtained from observations (X-rays, EUV, radio, infrared, ...) to derive a magnetic field configuration closer to reality, and thus to allow for quantitative comparison. As an example, the comparison between the density derived from MHS models and the density obtained from spectrometers should be a systematic check of the goodness of the extrapolation.

The main word for the development of extrapolation techniques using solar data as boundary conditions is quantitative. The main issue is always to find a compromise between the spatial resolution, the size of the field-of-view, the computational time, and the resources, which is the most suitable for the physical problem tackled.

\subsection{Comments on Boundary Conditions}

I briefly discuss the current issues encountered when using solar magnetograms as boundary conditions for magnetic field extrapolations. In the following, a "flat surface" refers to a surface of constant curvature or radius (e.g., a plane or a sphere). The main issues that should be kept in mind when reconstructing a coronal magnetic field are:

- Inversion of Stokes parameters: the magnetic field components are derived from the radiative transfer equations for the four Stokes parameters. The inversion is complex and often used by reconstruction modellers as a black box. One typical assumption is to consider that the solar atmosphere as a constant optical depth. This assumption with the assumption of a local thermodynamic equilibrium is becoming less and less accurate for high resolution data. 
- 180 degree ambiguity: after the inversion of the Stokes parameters, an ambiguity of 180 degrees on the orientation of the transverse magnetic field still remains. Some algorithms to resolve this ambiguity are based on a magnetic field model (potential, LFF, or NLFF field). The goodness of the ambiguity is crucial, especially when a flux rope is present in the magnetic configuration.

- Flatness of the surface: as implied by the inversion of the Stokes parameters, the measurement of the magnetic field in neighbouring pixels is certainly not at the same height in the photosphere. This fact is even more critical for chromospheric measurements. In order to currently perform a magnetic field extrapolation, it is assumed that the magnetogram corresponds to a flat surface.

- The $\alpha$-distribution: the definition of $\alpha$ as seen in Equation. (6) is given by the normal component of the force-free equation. It means that a contribution from the electric currents perpendicular to the magnetic field is included into the $\alpha$-distribution when the magnetic field is not strictly normal to the photospheric surface. The effects of the electric currents have to be investigated in more details. For extrapolation models beyond the force-free models, the components of the electric current density will be more appropriate boundary conditions (Grad and Rubin, 1958).

- Error estimate: it is still difficult to estimate the error/inacurracy made in measuring the magnetic field: (i) the signal in sunspot is weak (limited number of photons), (ii) the magnetic field in quiet Sun regions is weak, (iii) the Hanle effect can dominate the quiet-Sun field, (iv) the linear polarisation signal leading to the transverse magnetic field is small compared to the Stokes $V$ signal (by an order of magnitude), (v) the properties of the spectral line such as the Landé factor can influence the threshold/saturation of the detected magnetic field strength, (vi) a pixel is not filled uniformly by magnetic field, so the filling factor is also an important quantity. Error estimates from vector magnetograms can be incorporate in the extrapolation model resulting in a reliable equilibrium (Wheatland and Leka, 2011).

- Projection effect: the transverse field is strongly influenced by the projection effect, especially in the penumbra of sunspots where the magnetic field is mostly horizontal. This leads to the change of sign of observed polarities.

- Synoptic/Carrington maps: often by default, the Carrington maps have been corrected for the magnetic flux imbalance, which is good for extrapolation but do not represent correctly the physics of the solar surface magnetic field.

In the near future, we will see the development of new instruments which will provide us access to the magnetic field near the poles (e.g., Solar Orbiter) and in the corona (e.g., CoMP). These improved measurements will impose new constraints on the physics of magnetic field extrapolations.

\section{Summary}

In this review, I have summarised many different results obtained from magnetic field extrapolations combined with magnetic field observations. The extrapola- 
tions have been applied to various different issues in order to better understand the physics of the corona. Owing to the large number of observations in strong field regions, most of the studies have dealt with the structure and evolution of the magnetic field in active regions, as well as studying the causes and consequences of flares and CMEs.

What have we learned from magnetic field extrapolations?

- The current force-free models describe relatively well the structure of the coronal magnetic above active regions.

- The amount of magnetic energy available is consistent with what is expected and observed during eruptive events, even if most of time, the studies do not specify error bars.

- The existence of twisted flux bundles which are a prime ingredient in most of the MHD models to store magnetic energy in the corona, and trigger eruptions.

- The physics of flares is closely related to the topology of the magnetic field prior to the eruption: existence of coronal null points, separators or quasi-spearatrix layers.

- The large-scale connectivity of the magnetic field lines deduced from extrapolations is crucial to understanding of the redistribution of magnetic energy and magnetic helictiy in the solar corona.

- The link between the broad range of observations (from hard X-ray to radio wavelengths) can be made through the structure of the magnetic field.

What have we not learned (yet) from magnetic field extrapolations?

- The "universal" ingredient responsible for triggering eruptions and which will allow us to predict flares and CMEs.

- The interaction between the different spatial scales involved in the solar atmosphere, from the quiet-Sun magnetic field to the global structure.

- The interaction between the different regions of the Sun: from the tachocline to the solar wind, englobing the whole heliosphere.

- To be specific to a particular extrapolation method, we do not yet understand the goodness of the force-free extrapolations while the imposed boundary conditions are not force-free. To achieve the goal, it is required to develop more theoretical studies based on the effects of noise or perpendicular currents on force-free magnetic configurations.

Despite this apparent success of extrapolation methods, the improvement in the understanding of the coronal magnetic field will depend on quantitative comparisons with the observations and not just qualitative comparisons. The quantitative success of the extrapolation method will a posteriori justify the physical assumptions or will drive the developements of these methods towards more physical and sophisticated techniques. The limits of these developments are the boundary conditions obtained by the observations, and the computational power available. Especially, there is a need to improve the physics incorporated into these models: the coupling between the plasma and the magnetic field playing a major role in the evolution of the magnetic fields. 
Acknowledgements I would like to thank Steve Tomczyk and Marc DeRosa for inviting me to contribute to the "Coronal Magnetism - Connecting Models to Data and the Corona to the Earth" workshop held in Boulder (2012). I also thank Michael Thompson for his hospitality at HAO (Boulder) during the workshop.

\section{References}

Abramenko, V.I., Yurchishin, V.B.: 1996, Solar Phys. 168, 47. doi:10.1007/BF00145824.

Abramenko, V.I., Wang, T., Yurchishin, V.B.: 1996, Solar Phys. 168, 75. doi:10.1007/BF00145826.

Adams, J., Pneuman, G.W.: 1976, Solar Phys. 46, 185. doi:10.1007/BF00157566.

Aiouaz, T., Rast, M.P.: 2006, Astrophys. J. 647, 183. doi:10.1086/507468.

Alexander, C.E., Del Zanna, G., Maclean, R.C.: 2011, Astron. Astrophys. 526, 134. doi:10.1051/0004-6361/201014045.

Alissandrakis, C.E.: 1981, Astron. Astrophys. 100, 197.

Altschuler, M.D., Newkirk, G.: 1969, Solar Phys. 9, 131. doi:10.1007/BF00145734.

Aly, J.J.: 1984, Astrophys. J. 283, 349. doi:10.1086/162313.

Aly, J.J.: 1989, Solar Phys. 120, 19. doi:10.1007/BF00148533

Amari, T., Aly, J.J.: 2010, Astron. Astrophys. 522, 52. doi:10.1051/0004-6361/200913058.

Amari, T., Boulmezaoud, T.Z., Aly, J.J.: 2006, Astron. Astrophys. 446, 691. doi:10.1051/00046361:20054076.

Amari, T., Boulmezaoud, T.Z., Maday, Y.: 1998, Astron. Astrophys. 339, 252.

Amari, T., Boulmezaoud, T.Z., Mikic, Z.: 1999, Astron. Astrophys. 350, 1051.

Amari, T., Aly, J.J., Luciani, J.F., Boulmezaoud, T.Z., Mikic, Z.: 1997, Solar Phys. 174, 129.

Antiochos, S.K.: 1987, Astrophys. J. 312, 886. doi:10.1086/164935.

Arzner, K., Vlahos, L.: 2006, Astron. Astrophys. 454, 957. doi:10.1051/0004-6361:20064953.

Aschwanden, M.J.: 2005, Solar Phys. 228, 339. doi:10.1007/s11207-005-2788-5.

Aschwanden, M.J.: 2012a, Solar Phys.. doi:10.1007/s11207-012-0069-7.

Aschwanden, M.J.: 2012b, Solar Phys.. doi:10.1007/s11207-012-0203-6.

Aschwanden, M.J.: 2013, Astrophys. J. 763, 115. doi:10.1088/0004-637X/763/2/115.

Aschwanden, M.J., Malanushenko, A.: 2012, Solar Phys.. doi:10.1007/s11207-012-0070-1.

Aschwanden, M.J., Sandman, A.W.: 2010, Astron. J. 140, 723. doi:10.1088/0004$6256 / 140 / 3 / 723$

Aschwanden, M.J., Lee, J.K., Gary, G.A., Smith, M., Inhester, B.: 2008, Solar Phys. 248, 359. doi:10.1007/s11207-007-9064-9.

Aulanier, G., Démoulin, P.: 1998, Astron. Astrophys. 329, 1125.

Aulanier, G., Démoulin, P., van Driel-Gesztelyi, L., Mein, P., Deforest, C.: 1998, Astron. Astrophys. 335, 309.

Aulanier, G., Démoulin, P., Mein, N., van Driel-Gesztelyi, L., Mein, P., Schmieder, B.: 1999, Astron. Astrophys. 342, 867.

Aulanier, G., DeLuca, E.E., Antiochos, S.K., McMullen, R.A., Golub, L.: 2000, Astrophys. J. 540, 1126. doi:10.1086/309376.

Aurass, H., Klein, K.L., Zlotnik, E.Y., Zaitsev, V.V.: 2003, Astron. Astrophys. 410, 1001. doi:10.1051/0004-6361:20031249.

Aurass, H., Rausche, G., Mann, G., Hofmann, A.: 2005, Astron. Astrophys. 435, 1137. doi:10.1051/0004-6361:20042199.

Aurass, H., Rausche, G., Berkebile-Stoiser, S., Veronig, A.: 2010, Astron. Astrophys. 515, 1. doi:10.1051/0004-6361/200913132.

Aurass, H., Mann, G., Zlobec, P., Karlický, M.: 2011, Astrophys. J. 730, 57. doi:10.1088/0004$637 \times / 730 / 1 / 57$.

Baker, D., van Driel-Gesztelyi, L., Mandrini, C.H., Démoulin, binitsP., Murray, M.J.: 2009, Astrophys. J. 705, 926. doi:10.1088/0004-637X/705/1/926.

Barnes, G., Leka, K.D., Wheatland, M.S.: 2006, Astrophys. J. 641, 1188. doi:10.1086/500503.

Barnes, G., Longcope, D.W., Leka, K.D.: 2005, Astrophys. J. 629, 561. doi:10.1086/431175.

Bentley, R.D., Klein, K.L., van Driel-Gesztelyi, L., Démoulin, P., Trottet, G., Tassetto, P., Marty, G.: 2000, Solar Phys. 193, 227. doi:10.1023/A:1005218007132

Berger, M.A.: 1999a, Plasma Phys. Control. Fusion 41, 167. doi:10.1088/0741$3335 / 41 / 12 B / 312$ 
Berger, M.A.: 1999b, In: Brown, M.R., Canfield, R.C., Pevtsov, A.A. (eds.) Magnetic Helicity in Space and Laboratory Plasmas, AGU Geophys. Monogr., 111. doi:10.1029/GM111p0001.

Berger, M.A., Field, G.B.: 1984, J. Fluid Mech. 147, 133. doi:10.1017/S0022112084002019

Berger, M.A., Prior, C.: 2006, J. Physics A 39, 8321. doi:10.1088/0305-4470/39/26/005.

Berlicki, A., Schmieder, B., Vilmer, N., Aulanier, G., Del Zanna, G.: 2004, Astron. Astrophys. 423, 1119. doi:10.1051/0004-6361:20040259.

Bernasconi, P.N., Rust, D.M., Eaton, H.A.C.: 2001, In: Sigwarth, M. (ed.) Advanced Solar Polarimetry - Theory, Observation, and Instrumentation, ASP Conf. Ser. 236, 399.

Beveridge, C., Priest, E.R., Brown, D.S.: 2002, Solar Phys. 209, 333. doi:10.1023/A:1021210127598.

Beveridge, C., Priest, E.R., Brown, D.S.: 2004, Geophys. Astrophys. Fluid Dyn. 98, 429. doi:10.1080/03091920410001715575.

Bhattacharyya, R., Janaki, M.S., Dasgupta, B., Zank, G.P.: 2007, Solar Phys. 240, 63. doi:10.1007/s11207-006-0280-5.

Bogod, V.M., Stupishin, A.G., Yasnov, L.V.: 2012, Solar Phys. 276, 61. doi:10.1007/s11207011-9850-2.

Boulbe, C., Zamène Boulmezaoud, T., Amari, T.: 2009, ArXiv e-prints.

Boutry, C., Buchlin, E., Vial, J.C., Régnier, S.: 2012, Astrophys. J. 752, 13. doi:10.1088/0004$637 \mathrm{X} / 752 / 1 / 13$.

Brooks, D.H., Warren, H.P.: 2008, Astrophys. J. 687, 1363. doi:10.1086/591834.

Brosius, J.W., Davila, J.M., Thomas, R.J., White, S.M.: 1997, Astrophys. J. 488, 488. doi: $10.1086 / 304673$.

Brosius, J.W., Landi, E., Cook, J.W., Newmark, J.S., Gopalswamy, N., Lara, A.: 2002, Astrophys. J. 574, 453. doi:10.1086/340923.

Brown, D.S., Priest, E.R.: 1999, Proc. Roy. Soc. London A 455, 3931. doi:10.1098/rspa.1999.0484.

Brown, D.S., Priest, E.R.: 2000, Solar Phys. 194, 197. doi:10.1023/A:1005221503925

Bungey, T.N., Titov, V.S., Priest, E.R.: 1996, Astron. Astrophys. 308, 233.

Canfield, R.C., Hudson, H.S., McKenzie, D.E.: 1999, Geophys. Res. Lett. 26, 627. doi:10.1029/1999GL900105.

Canou, A., Amari, T., Bommier, V., Schmieder, B., Aulanier, G., Li, H.: 2009, Astrophys. J. Lett. 693, L27. doi:10.1088/0004-637X/693/1/L27.

Canou, A., Amari, T.: 2010, Astrophys. J. 715, 1566. doi:10.1088/0004-637X/715/2/1566.

Carcedo, L., Brown, D.S., Hood, A.W., Neukirch, T., Wiegelmann, T.: 2003, Solar Phys. 218, 29. doi:10.1023/B:SOLA.0000013045.65499.da.

Chandra, R., Schmieder, B., Aulanier, G., Malherbe, J.M.: 2009, Solar Phys. 258, 53. doi:10.1007/s11207-009-9392-z.

Chen, B., Bastian, T.S., Gary, D.E., Jing, J.: 2011, Astrophys. J. 736, 64. doi:10.1088/0004$637 X / 736 / 1 / 64$.

Cheng, X., Ding, M.D., Guo, Y., Zhang, J., Jing, J., Wiegelmann, T.: 2010, Astrophys. J. Lett. 716, 68. doi:10.1088/2041-8205/716/1/L68.

Cheng, X., Zhang, J., Ding, M.D., Guo, Y., Su, J.T.: 2011, Astrophys. J. 732, 87. doi:10.1088/0004-637X/732/2/87.

Chiu, Y.T., Hilton, H.H.: 1977, Astrophys. J. 212, 873. doi:10.1086/155111.

Choudhary, D.P., Gary, G.A.: 1999, Solar Phys. 188, 345. doi:10.1023/A:1005165425964

Clegg, J.R., Bromage, B.J.I., Browning, P.K.: 1999, Space Sci. Rev. 87, 145. doi:10.1023/A:1005190821498.

Clegg, J.R., Browning, P.K., Laurence, P., Bromage, B.J.I., Stredulinsky, E.: 2000, Astron. Astrophys. 361, 743.

Conlon, P.A., Gallagher, P.T.: 2010, Astrophys. J. 715, 59. doi:10.1088/0004-637X/715/1/59.

Contopoulos, I.: 2013, Solar Phys. 282, 419. doi:10.1007/s11207-012-0154-y.

Contopoulos, I., Kalapotharakos, C., Georgoulis, M.K.: 2011, Solar Phys. 269, 351. doi:10.1007/s11207-011-9713-x.

Courant, Hilbert: 1963, J. Appl. Mech. 30, 158. doi:10.1115/1.3630089.

Cuperman, S., Ofman, L., Semel, M.: 1989, Astron. Astrophys. 216, 265.

Cuperman, S., Ofman, L., Semel, M.: 1990a, Astron. Astrophys. 227, 583.

Cuperman, S., Ofman, L., Semel, M.: 1990b, Astron. Astrophys. 230, 193.

De Rosa, M.L., Schrijver, C.J., Barnes, G., Leka, K.D., Lites, B.W., Aschwanden, M.J., Amari, T., Canou, A., McTiernan, J.M., Régnier, S., Thalmann, J.K., Valori, G., Wheatland, M.S., 
Wiegelmann, T., Cheung, M.C.M., Conlon, P.A., Fuhrmann, M., Inhester, B., Tadesse, T.: 2009, Astrophys. J. 696, 1780. doi:10.1088/0004-637X/696/2/1780.

DeForest, C.E., Hagenaar, H.J., Lamb, D.A., Parnell, C.E., Welsch, B.T.: 2007, Astrophys. J. 666, 576. doi:10.1086/518994.

Del Zanna, G., Berlicki, A., Schmieder, B., Mason, H.E.: 2006, Solar Phys. 234, 95. doi:10.1007/s11207-006-0016-6.

Del Zanna, G., Aulanier, G., Klein, K.L., Török, T.: 2011, Astron. Astrophys. 526, 137. doi:10.1051/0004-6361/201015231.

Delannée, C., Aulanier, G.: 1999, Solar Phys. 190, 107. doi:10.1023/A:1005249416605.

Démoulin, P., Cuperman, S., Semel, M.: 1992, Astron. Astrophys. 263, 351.

Démoulin, P., Mandrini, C.H., Rovira, M.G., Hénoux, J.C., Machado, M.E.: 1994, Solar Phys. 150, 221. doi:10.1007/BF00712887.

des Jardins, A., Canfield, R., Longcope, D., McLinden, E., Dillman, A.: 2009, Astrophys. J. 693, 886. doi:10.1088/0004-637X/693/1/886.

Dudík, J., Aulanier, G., Schmieder, B., Bommier, V., Roudier, T.: 2008, Solar Phys. $248,29$. doi:10.1007/s11207-008-9155-2.

Durrant, C.J.: 1989, Aust. J. Phys. 42, 317.

Finn, J.M., Antonsen, T.M.: 1985, Comments Plasma Phys. Controlled Fusion 9, 111.

Fragos, T., Rantsiou, E., Vlahos, L.: 2004, Astron. Astrophys. 420, 719. doi:10.1051/00046361:20034570.

Fuhrmann, M., Seehafer, N., Valori, G., Wiegelmann, T.: 2011, Astron. Astrophys. 526, 70. doi:10.1051/0004-6361/201015453.

Gary, G.A.: 1989, Astrophys. J. Suppl. 69, 323. doi:10.1086/191316.

Gary, G.A., Alexander, D.: 1999, Solar Phys. 186, 123. doi:10.1023/A:1005147921110.

Gary, G.A., Moore, R.L.: 2004, Astrophys. J. 611, 545. doi:10.1086/422132.

Georgoulis, M.K., Tziotziou, K., Raouafi, N.E.: 2012, Astrophys. J. 759, 1. doi:10.1088/0004$637 \mathrm{X} / 759 / 1 / 1$.

Gilchrist, S.A., Wheatland, M.S.: 2013, Solar Phys. 282, 283. doi:10.1007/s11207-012-0144-0.

Gilchrist, S.A., Wheatland, M.S., Leka, K.D.: 2012, Solar Phys. 276, 133. doi:10.1007/s11207011-9878-3

Gold, T., Hoyle, F.: 1960, Mon. Not. Roy. Astron. Soc. 120, 89.

Golub, L., Krieger, A.S., Silk, J.K., Timothy, A.F., Vaiana, G.S.: 1974, Astrophys. J. Lett. 189, L93. doi:10.1086/181472.

Goodman, M.L.: 2000, Astrophys. J. 533, 501. doi:10.1086/308635

Grad, H., Rubin, H.: 1958, In: Proc. 2nd Int. Conf. on Peaceful Uses of Atomic Energy, 31, 190.

Grebinskij, A., Bogod, V., Gelfreikh, G., Urpo, S., Pohjolainen, S., Shibasaki, K.: 2000, Astron. Astrophys. Suppl. 144, 169. doi:10.1051/aas:2000202.

Grechnev, V.V., Uralov, A.M., Zandanov, V.G., Rudenko, G.V., Borovik, V.N., Grigorieva, I.Y., Slemzin, V.A., Bogachev, S.A., Kuzin, S.V., Zhitnik, I., Pertsov, A.A., Shibasaki, K., Livshits, M.A.: 2006, Publ. Astron. Soc. Japan 58, 55.

Guglielmino, S.L., Bellot Rubio, L.R., Zuccarello, F., Aulanier, G., Vargas Domínguez, S., Kamio, S.: 2010, Astrophys. J. 724, 1083. doi:10.1088/0004-637X/724/2/1083.

Guo, Y., Ding, M.D., Wiegelmann, T., Li, H.: 2008, Astrophys. J. 679, 1629. doi: $10.1086 / 587684$.

Guo, Y., Schmieder, B., Démoulin, P., Wiegelmann, T., Aulanier, G., Török, T., Bommier, V.: 2010a, Astrophys. J. 714, 343. doi:10.1088/0004-637X/714/1/343.

Guo, Y., Ding, M.D., Schmieder, B., Li, H., Török, T., Wiegelmann, T.: 2010b, Astrophys. J. Lett. 725, 38. doi:10.1088/2041-8205/725/1/L38.

Guo, Y., Ding, M.D., Schmieder, B., Démoulin, P., Li, H.: 2012, Astrophys. J. 746, 17. doi:10.1088/0004-637X/746/1/17.

Hakamada, K.: 1995, Solar Phys. 159, 89. doi:10.1007/BF00733033.

Hao, Q., Guo, Y., Dai, Y., Ding, M.D., Li, Z., Zhang, X.Y., Fang, C.: 2012, Astron. Astrophys. 544, 17. doi:10.1051/0004-6361/201219941.

He, H., Wang, H.: 2006, Mon. Not. Roy. Astron. Soc. 369, 207. doi:10.1111/j.13652966.2006.10288.x

He, H., Wang, H.: 2008, J. Geophys. Res. 113, 5. doi:10.1029/2007JA012441.

He, J.S., Marsch, E., Curdt, W., Tian, H., Tu, C.Y., Xia, L.D., Kamio, S.: 2010a, Astron. Astrophys. 519, 49. doi:10.1051/0004-6361/201014709.

He, J.S., Marsch, E., Tu, C.Y., Tian, H., Guo, L.J.: 2010b, Astron. Astrophys. 510, 40. doi:10.1051/0004-6361/200913059 
Heyvaerts, J., Priest, E.R.: 1984, Astron. Astrophys. 137, 63.

Hildebrandt, J., Seehafer, N., Krueger, A.: 1984, Astron. Astrophys. 134, 185.

Hofmann, A., Ruždjak, V.: 2007, Solar Phys. 240, 107. doi:10.1007/s11207-006-0228-9.

Hu, Q., Dasgupta, B.: 2006, Geophys. Res. Lett. 33, 15106. doi:10.1029/2006GL026952.

Hu, Q., Dasgupta, B.: 2008, Solar Phys. 247, 87. doi:10.1007/s11207-007-9090-7.

Hu, Q., Dasgupta, B., Choudhary, D.P., Büchner, J.: 2008, Astrophys. J. 679, 848. doi:10.1086/587639.

Hu, Q., Dasgupta, B., Derosa, M.L., Büchner, J., Gary, G.A.: 2010, J. Atmos. and Solar-Terr. Phys. 72, 219. doi:10.1016/j.jastp.2009.11.014.

Hudson, T.S., Wheatland, M.S.: 1999, Solar Phys. 186, 301. doi:10.1023/A:1005176500346.

Inhester, B., Wiegelmann, T.: 2006, Solar Phys. 235, 201. doi:10.1007/s11207-006-0065-x.

Inhester, B., Feng, L., Wiegelmann, T.: 2008, Solar Phys. 248, 379. doi:10.1007/s11207-0079027-1.

Inoue, S., Kusano, K., Magara, T., Shiota, D., Yamamoto, T.T.: 2011, Astrophys. J. 738, 161. doi:10.1088/0004-637X/738/2/161

Inoue, S., Magara, T., Watari, S., Choe, G.S.: 2012, Astrophys. J. 747, 65. doi:10.1088/0004$637 \mathrm{X} / 747 / 1 / 65$

Iwai, K., Miyoshi, Y., Masuda, S., Shimojo, M., Shiota, D., Inoue, S., Tsuchiya, F., Morioka, A., Misawa, H.: 2012, Astrophys. J. 744, 167. doi:10.1088/0004-637X/744/2/167.

Jiang, C., Feng, X., Fan, Y., Xiang, C.: 2011, Astrophys. J. 727, 101. doi:10.1088/0004$637 \mathrm{X} / 727 / 2 / 101$.

Jiao, L., McClymont, A.N., Mikic, Z.: $1997, \quad$ Solar Phys. 174, 311. doi:10.1023/A:1004927101300.

Jin, C., Wang, J.: 2011, Astrophys. J. 732, 4. doi:10.1088/0004-637X/732/1/4.

Jing, J., Wiegelmann, T., Suematsu, Y., Kubo, M., Wang, H.: 2008, Astrophys. J. Lett. 676, L81. doi:10.1086/587058.

Jing, J., Chen, P.F., Wiegelmann, T., Xu, Y., Park, S.H., Wang, H.: 2009, Astrophys. J. 696, 84. doi:10.1088/0004-637X/696/1/84.

Jing, J., Tan, C., Yuan, Y., Wang, B., Wiegelmann, T., Xu, Y., Wang, H.: 2010, Astrophys. J. 713, 440. doi:10.1088/0004-637X/713/1/440.

Jing, J., Yuan, Y., Reardon, K., Wiegelmann, T., Xu, Y., Wang, H.: 2011, Astrophys. J. 739, 67. doi:10.1088/0004-637X/739/2/67

Jing, J., Park, S.H., Liu, C., Lee, J., Wiegelmann, T., Xu, Y., Deng, N., Wang, H.: 2012, Astrophys. J. Lett. 752, L9. doi:10.1088/2041-8205/752/1/L9.

Klassen, A., Aurass, H., Klein, K.L., Hofmann, A., Mann, G.: 1999, Astron. Astrophys. 343, 287.

Klein, K.L., Krucker, S., Lointier, G., Kerdraon, A.: 2008, Astron. Astrophys. 486, 589. doi:10.1051/0004-6361:20079228.

Klimchuk, J.A., Sturrock, P.A.: 1992, Astrophys. J. 385, 344. doi:10.1086/170943.

Kusano, K., Nishikawa, K.: 1996, Astrophys. J. 461, 415. doi:10.1086/177070.

Kwon, R.Y., Chae, J.: 2008, Astrophys. J. Lett. 677, L141. doi:10.1086/587981.

Lamb, D.A., DeForest, C.E., Hagenaar, H.J., Parnell, C.E., Welsch, B.T.: 2008, Astrophys. J. 674, 520. doi:10.1086/524372.

Lamb, D.A., DeForest, C.E., Hagenaar, H.J., Parnell, C.E., Welsch, B.T.: 2010, Astrophys. J. 720, 1405. doi:10.1088/0004-637X/720/2/1405.

Lee, J., McClymont, A.N., Mikic, Z., White, S.M., Kundu, M.R.: 1998, Astrophys. J. 501, 853. doi:10.1086/305851.

Lee, J., White, S.M., Kundu, M.R., Mikić, Z., McClymont, A.N.: 1999, Astrophys. J. 510, 413. doi:10.1086/306556.

Leka, K.D.: 1996, Astrophys. J. 462, 547. doi:10.1086/177171.

Leka, K.D.: 1999, Solar Phys. 188, 21. doi:10.1023/A:1005130630873.

Leka, K.D., Skumanich, A.: 1999, Solar Phys. 188, 3. doi:10.1023/A:1005108632671.

Levine, R.H., Altschuler, M.D.: 1974, Solar Phys. 36, 345. doi:10.1007/BF00151204.

Levine, R.H., Schulz, M., Frazier, E.N.: 1982, Solar Phys. 77, 363. doi:10.1007/BF00156118.

Li, H., Schmieder, B., Song, M.T., Bommier, V.: 2007, Astron. Astrophys. 475, 1081. doi:10.1051/0004-6361:20077500.

Li, Y., Song, G., Li, J.: 2009, Solar Phys. 260, 109. doi:10.1007/s11207-009-9431-9.

Li, Z., Yan, Y., Song, G.: 2004, Mon. Not. Roy. Astron. Soc. 347, 1255. doi:10.1111/j.13652966.2004.07309.x

Lin, Y.: 1990, Comput. Phys. Commun. 59, 139. doi:10.1016/0010-4655(90)90163-U.

Lin, Y.Z., Wang, Z.Z., Wu, F., Ding, Y.J., Hong, Q.F.: 1985, Acta Astrophys. Sinica 5, 19. 
Linke, J., Ioshpa, B.A., Selivanov, V.L.: 1990, Astron. Nachr. 311, 309.

Liu, C., Lee, J., Jing, J., Liu, R., Deng, N., Wang, H.: 2010, Astrophys. J. Lett. 721, L193. doi:10.1088/2041-8205/721/2/L193

Liu, S., Zhang, H.Q., Su, J.T., Song, M.T.: 2011, Solar Phys. 269, 41. doi:10.1007/s11207010-9691-4.

Longcope, D.W.: 2005, Living Reviews in Solar Physics 2 (7). doi:10.12942/Irsp-2005-7. http://solarphysics.livingreviews.org/Articles/Irsp-2005-7/

Longcope, D.W., Des Jardins, A.C., Carranza-Fulmer, T., Qiu, J.: 2010, Solar Phys. 267, 107. doi:10.1007/s11207-010-9635-z.

Longcope, D.W., Klapper, I.: 2002, Astrophys. J. 579, 468. doi:10.1086/342750

Longcope, D.W., Malanushenko, A.: 2008, Astrophys. J. 674, 1130. doi:10.1086/524011.

Ma, E., Ai, G.X.: 1979, Acta Astron. Sinica 20, 374.

Mackay, D., Yeates, A.: 2012, Living Rev. Solar Phys. 6 (9). doi:10.12942/Irsp-2012-6. http://solarphysics.livingreviews.org/Articles/Irsp-2012-6/

Maclean, R.C., Beveridge, C., Priest, E.R.: 2006, Solar Phys. 238, 13. doi:10.1007/s11207-0060179-1.

Maclean, R.C., Beveridge, C., Hornig, G., Priest, E.R.: 2006, Solar Phys. 237, 227. doi:10.1007/s11207-006-9002-2.

Malanushenko, A., Schrijver, C.J., DeRosa, M.L., Wheatland, M.S., Gilchrist, S.A.: 2012, Astrophys. J. 756, 153. doi:10.1088/0004-637X/756/2/153.

Malanushenko, A., Longcope, D.W., McKenzie, D.E.: 2009, Astrophys. J. 707, 1044. doi:10.1088/0004-637X/707/2/1044.

Mandrini, C.H., Demoulin, P., Rovira, M.G., de La Beaujardiere, J.F., Henoux, J.C.: 1995, Astron. Astrophys. 303, 927.

Mandrini, C.H., Démoulin, P., van Driel-Gesztelyi, L., Schmieder, B., Cauzzi, G., Hofmann, A.: 1996, Solar Phys. 168, 115. doi:10.1007/BF00145829.

Martínez-Oliveros, J.C., Moradi, H., Donea, A.C.: 2008, Solar Phys. 251, 613. doi:10.1007/s11207-008-9122-y.

Masson, S., Pariat, E., Aulanier, G., Schrijver, C.J.: 2009, Astrophys. J. 700, 559. doi:10.1088/0004-637X/700/1/559.

McClymont, A.N., Mikic, Z.: 1994, Astrophys. J. 422, 899. doi:10.1086/173781.

Metcalf, T.R., De Rosa, M.L., Schrijver, C.J., Barnes, G., van Ballegooijen, A.A., Wiegelmann, T., Wheatland, M.S., Valori, G., McTtiernan, J.M.: 2008, Solar Phys. 247, 269. doi:10.1007/s11207-007-9110-7.

Meyer, K.A., Mackay, D.H., van Ballegooijen, A.A.: 2012, Solar Phys. 278, 149. doi:10.1007/s11207-011-9924-1.

Meyer, K.A., Mackay, D.H., van Ballegooijen, A.A., Parnell, C.E.: 2011, Solar Phys. 272, 29. doi:10.1007/s11207-011-9809-3.

Mikic, Z., Barnes, D.C., Schnack, D.D.: 1988, Astrophys. J. 328, 830. doi:10.1086/166341.

Molodensky, M.M.: 1974, Solar Phys. 39, 393. doi:10.1007/BF00162432.

Moon, Y.J., Chae, J., Choe, G.S., Wang, H., Park, Y.D., Cheng, C.Z.: 2004, J. Korean Astron. Soc. 37, 41 .

Mysh'yakov, I.I., Rudenko, G.V.: 2009, Geomag. Aeron. 49, 940. doi:10.1134/S0016793209070184.

Nakagawa, Y., Raadu, M.A.: 1972, Solar Phys. 25, 127. doi:10.1007/BF00155751.

Nakagawa, Y., Raadu, M.A., Harvey, J.W.: 1973, Solar Phys. 30, 421. doi:10.1007/BF00152673.

Nakagawa, Y., Wu, S.T., Tandberg-Hanssen, E.: 1978, Astron. Astrophys. 69, 43.

Nitta, N.V., De Rosa, M.L.: 2008, Astrophys. J. Lett. 673, L207. doi:10.1086/527548.

Paesold, G., Benz, A.O., Klein, K.L., Vilmer, N.: 2001, Astron. Astrophys. 371, 333. doi:10.1051/0004-6361:20010358.

Paletou, F., López Ariste, A., Bommier, V., Semel, M.: 2001, Astron. Astrophys. 375, 39. doi:10.1051/0004-6361:20010927.

Pallavicini, R., Sakurai, T., Vaiana, G.S.: 1981, Astron. Astrophys. 98, 316.

Pariat, E., Aulanier, G., Schmieder, B., Georgoulis, M.K., Rust, D.M., Bernasconi, P.N.: 2004, Astrophys. J. 614, 1099. doi:10.1086/423891.

Park, S.H., Chae, J., Jing, J., Tan, C., Wang, H.: 2010, Astrophys. J. 720, 1102. doi:10.1088/0004-637X/720/2/1102

Priest, E.R., Longcope, D.W., Heyvaerts, J.: 2005, Astrophys. J. 624, 1057. doi:10.1086/429312.

Prior, C., Berger, M.A.: 2012, Solar Phys. 278, 323. doi:10.1007/s11207-012-9938-3. 
Régnier, S.: 2012, Solar Phys. 277, 131. doi:10.1007/s11207-011-9830-6.

Régnier, S., Amari, T.: 2004, Astron. Astrophys. 425, 345. doi:10.1051/0004-6361:20034383.

Régnier, S., Canfield, R.C.: 2006, Astron. Astrophys. 451, 319. doi:10.1051/00046361:20054171.

Régnier, S., Priest, E.R.: 2007a, Astron. Astrophys. 468, 701. doi:10.1051/00046361:20077318

Régnier, S., Priest, E.R.: 2007b, Astrophys. J. 669, 53. doi:10.1086/523269.

Régnier, S., Amari, T., Canfield, R.C.: 2005, Astron. Astrophys. 442, 345. doi:10.1051/00046361:20053509

Régnier, S., Amari, T., Kersalé, E.: 2002, Astron. Astrophys. 392, 1119. doi:10.1051/00046361:20020993.

Régnier, S., Parnell, C.E., Haynes, A.L.: 2008, Astron. Astrophys. 484, 47. doi:10.1051/00046361:200809826.

Régnier, S., Priest, E.R., Hood, A.W.: 2008, Astron. Astrophys. 491, 297. doi:10.1051/00046361:200810362

Roumeliotis, G.: 1996, Astrophys. J. 473, 1095. doi:10.1086/178219.

Rudenko, G.V.: 2001, Solar Phys. 198, 5. doi:10.1023/A:1005270431628.

Rudenko, G.V., Myshyakov, I.I.: 2009, Solar Phys. 257, 287. doi:10.1007/s11207-009-9389-7.

Rust, D.M., Nakagawa, Y., Neupert, W.M.: 1975, Solar Phys. 41, 397. doi:10.1007/BF00154077.

Ryabov, B.I., Maksimov, V.P., Lesovoi, S.V., Shibasaki, K., Nindos, A., Pevtsov, A.: 2005, Solar Phys. 226, 223. doi:10.1007/s11207-005-2691-0.

Sakurai, T.: 1981, Solar Phys. 69, 343. doi:10.1007/BF00149999.

Sakurai, T.: 1985, Solar Phys. 95, 311. doi:10.1007/BF00152408.

Sandman, A.W., Aschwanden, M.J., Derosa, M.L., Wülser, J.P., Alexander, D.: 2009, Solar Phys. 259, 1. doi:10.1007/s11207-009-9383-0.

Savcheva, A.S., van Ballegooijen, A.A., DeLuca, E.E.: 2012, Astrophys. J. 744, 78. doi:10.1088/0004-637X/744/1/78.

Savcheva, A., Pariat, E., van Ballegooijen, A., Aulanier, G., DeLuca, E.: 2012, Astrophys. J. 750, 15. doi:10.1088/0004-637X/750/1/15

Schatten, K.H., Wilcox, J.M., Ness, N.F.: 1969, Solar Phys. 6, 442. doi:10.1007/BF00146478.

Schmahl, E.J., Kundu, M.R., Strong, K.T., Bentley, R.D., Smith, J.B. Jr., Krall, K.R.: 1982, Solar Phys. 80, 233. doi:10.1007/BF00147971.

Schmidt, H.U.: 1964, In: Hess, W.N. (ed.) The Physics of Solar Flares, NASA SP-50, 107

Schnack, D.D., Mikić, Z., Barnes, D.C., van Hoven, G.: 1990, Comput. Phys. Commun. 59, 21. doi:10.1016/0010-4655(90)90153-R.

Schrijver, C.J., Title, A.M.: 2002, Solar Phys. 207, 223. doi:10.123/A:1016295516408.

Schrijver, C.J., De Rosa, M.L., Metcalf, T.R., Liu, Y., McTiernan, J., Régnier, S., Valori, G., Wheatland, M.S., Wiegelmann, T.: 2006, Solar Phys. 235, 161. doi:10.1007/s11207-0060068-7.

Schrijver, C.J., De Rosa, M.L., Metcalf, T., Barnes, G., Lites, B., Tarbell, T., McTiernan, J., Valori, G., Wiegelmann, T., Wheatland, M.S., Amari, T., Aulanier, G., Démoulin, P., Fuhrmann, M., Kusano, K., Régnier, S., Thalmann, J.K.: 2008, Astrophys. J. 675, 1637. doi:10.1086/527413.

Seehafer, N.: 1975, Astron. Nachr. 296, 177.

Seehafer, N.: 1982, Solar Phys. 81, 69. doi:10.1007/BF00151981.

Seehafer, N.: 1985, Solar Phys. 96, 307. doi:10.1007/BF00149686.

Seehafer, N., Staude, J.: 1979, Astron. Nachr. 300, 151.

Semel, M.: 1988, Astron. Astrophys. 198, 293.

Shen, Y., Liu, Y., Su, J.: 2012, Astrophys. J. 750, 12. doi:10.1088/0004-637X/750/1/12.

Song, M.T., Fang, C., Tang, Y.H., Wu, S.T., Zhang, Y.A.: 2006, Astrophys. J. 649, 1084. doi:10.1086/506249.

Song, M.T., Fang, C., Zhang, H.Q., Tang, Y.H., Wu, S.T., Zhang, Y.A.: 2007, Astrophys. J. 666, 491. doi:10.1086/519829.

Sturrock, P.A.: 1991, Astrophys. J. 380, 655. doi:10.1086/170620.

Su, Q.R.: 1980, Acta Astron. Sinica 21, 152

Su, Q.R.: 1982, Solar Phys. 75, 229. doi:10.1007/BF00153473.

Su, Y., van Ballegooijen, A., Schmieder, B., Berlicki, A., Guo, Y., Golub, L., Huang, G.: 2009a, Astrophys. J. 704, 341. doi:10.1088/0004-637X/704/1/341.

Su, Y., van Ballegooijen, A., Lites, B.W., Deluca, E.E., Golub, L., Grigis, P.C., Huang, G., Ji, H.: 2009b, Astrophys. J. 691, 105. doi:10.1088/0004-637X/691/1/105. 
Sun, X., Hoeksema, J.T., Liu, Y., Wiegelmann, T., Hayashi, K., Chen, Q., Thalmann, J.: 2012, Astrophys. J. 748, 77. doi:10.1088/0004-637X/748/2/77.

Syntelis, P., Gontikakis, C., Georgoulis, M.K., Alissandrakis, C.E., Tsinganos, K.: 2012, Solar Phys. 280, 475. doi:10.1007/s11207-012-0026-5.

Tadesse, T., Wiegelmann, T., Inhester, B.: 2009, Astron. Astrophys. 508, 421. doi:10.1051/0004-6361/200912529.

Tadesse, T., Wiegelmann, T., Inhester, B., Pevtsov, A.: 2012, Solar Phys. 281, 53. doi:10.1007/s11207-012-9961-4

Takakura, T., Tsuneta, S., Nitta, N., Ohki, K.: 1983, Solar Phys. 86, 323. doi:10.1007/BF00157205.

Tanaka, K.: 1978, Solar Phys. 58, 149. doi:10.1007/BF00152561.

Tanaka, K., Nakagawa, Y.: 1973, Solar Phys. 33, 187. doi:10.1007/BF00152390.

Taylor, J.B.: 1974, Phys. Rev. Letters, 33, 1139. doi:10.1103/PhysRevLett.33.1139.

Thalmann, J.K., Wiegelmann, T.: 2008, Astron. Astrophys. 484, 495. doi:10.1051/00046361:200809508.

Thalmann, J.K., Wiegelmann, T., Raouafi, N.E.: 2008, Astron. Astrophys. 488, 71. doi:10.1051/0004-6361:200810235.

Titov, V.S.: 2007, Astrophys. J. 660, 863 doi:10.1086/512671.

Titov, V.S., Priest, E.R., Demoulin, P.: 1993, Astron. Astrophys. 276, 564.

Tun, S.D., Gary, D.E., Georgoulis, M.K.: 2011, Astrophys. J. 728, 1. doi:10.1088/0004$637 \mathrm{X} / 728 / 1 / 1$

Valori, G., Kliem, B., Fuhrmann, M.: 2007, Solar Phys. 245, 263. doi:10.1007/s11207-0079046-y.

Valori, G., Kliem, B., Keppens, R.: 2005, Astron. Astrophys. 433, 335. doi:10.1051/00046361:20042008

Valori, G., Kliem, B., Török, T., Titov, V.S.: 2010, Astron. Astrophys. 519, 44. doi:10.1051/0004-6361/201014416.

van Ballegooijen, A.A.: 2004, Astrophys. J. 612, 519. doi:10.1086/422512.

van Ballegooijen, A.A., Deluca, E.E., Squires, K., Mackay, D.H.: 2007, J. Atmos. Solar-Terr. Phys. 69, 24. doi:10.1016/j.jastp.2006.06.007.

Vincent, A., Charbonneau, P., Dubé, C.: 2012, Solar Phys. 278, 367. doi:10.1007/s11207-0129953-4.

Wang, D., Wei, F., Yan, Y.: 1995, Acta Astrophys. Sinica 15, 359.

Wang, H., Jing, J., Tan, C., Wiegelmann, T., Kubo, M.: 2008, Astrophys. J. 687, 658. doi:10.1086/592082

Wang, R., Tan, B., Tan, C., Yan, Y.: 2012, Solar Phys. 278, 411. doi:10.1007/s11207-012-99374.

Wang, T., Yan, Y., Wang, J., Kurokawa, H., Shibata, K.: 2002, Astrophys. J. 572, 580. doi:10.1086/340189.

Wang, Y.M.: 2000, Astrophys. J. Lett. 543, L89. doi:10.1086/318178.

Wen, Y.Y., Wang, J.X., Zhang, Y.Z.: 2007, Chin. J. Astron. Astrophys. 7, 265. doi:10.1088/1009-9271/7/2/11

Wheatland, M.S.: 2004, Solar Phys. 222, 247. doi:10.1023/B:SOLA.0000043579.93988.6f.

Wheatland, M.S.: 2006, Solar Phys. 238, 29. doi:10.1007/s11207-006-0232-0.

Wheatland, M.S., Leka, K.D.: 2011, Astrophys. J. 728, 112 . doi:10.1088/0004$637 \times / 728 / 2 / 112$.

Wheatland, M.S., Régnier, S.: 2009, Astrophys. J. Lett. 700, L88. doi:10.1088/0004$637 \mathrm{X} / 700 / 2 /$ L88.

Wheatland, M.S., Sturrock, P.A., Roumeliotis, G.: 2000, Astrophys. J. 540, 1150. doi:10.1086/309355.

Wiegelmann, T.: 2004, Solar Phys. 219, 87. doi:10.1023/B:SOLA.0000021799.39465.36.

Wiegelmann, T., Inhester, B.: 2010, Astron. Astrophys. 516, 107. doi:10.1051/0004$6361 / 201014391$.

Wiegelmann, T., Neukirch, T.: 2002, Solar Phys. 208, 233. doi:10.1023/A:1020537403934.

Wiegelmann, T., Neukirch, T.: 2006, Astron. Astrophys. 457, 1053. doi:10.1051/00046361:20065281.

Wiegelmann, T., Sakurai, T.: 2012, Living Rev. Solar Phys. 9 (5). doi:10.12942/Irsp-2012-5. http://solarphysics.livingreviews.org/Articles/Irsp-2012-5/

Wiegelmann, T., Inhester, B., Sakurai, T.: 2006, Solar Phys. 233, 215. doi:10.1007/s11207006-2092-z. 
Wiegelmann, T., Inhester, B., Lagg, A., Solanki, S.K.: 2005, Solar Phys. 228, 67. doi:10.1007/s11207-005-2511-6.

Wiegelmann, T., Neukirch, T., Ruan, P., Inhester, B.: 2007, Astron. Astrophys. 475, 701. doi:10.1051/0004-6361:20078244

Wiegelmann, T., Thalmann, J.K., Schrijver, C.J., De Rosa, M.L., Metcalf, T.R.: 2008, Solar Phys. 247, 249. doi:10.1007/s11207-008-9130-y.

Wiegelmann, T., Yelles Chaouche, L., Solanki, S.K., Lagg, A.: 2010, Astron. Astrophys. 511, 4. doi:10.1051/0004-6361/200912812.

Winebarger, A.R., Warren, H.P., Falconer, D.A.: 2008, Astrophys. J. 676, 672. doi:10.1086/527291.

Woltjer, L.: 1958, Proc. Natl. Acad. Sci. 44, 489.

Woodard, M.F., Chae, J.: 1999, Solar Phys. 184, 239. doi:10.1023/A:1005088232234.

Wu, F., Wang, Z.Z.: 1984, Acta Astrophys. Sinica 4, 272.

Wu, F., Wang, Z.Z.: 1985, Chin. J. Astron. Astrophys. 9, 44. doi:10.1016/0275-1062(85)90057-

Wu, S.T., Sun, M.T., Chang, H.M., Hagyard, M.J., Gary, G.A.: 1990, Astrophys. J. 362, 698. doi:10.1086/169307.

Xu, Y., Jing, J., Cao, W., Wang, H.: 2010, Astrophys. J. Lett. 709, L142. doi:10.1088/20418205/709/2/L142.

Yan, Y.: 1995, Solar Phys. 159, 97. doi:10.1007/BF00733034.

Yan, Y., Huang, G.: 2003, Space Sci. Rev. 107, 111. doi:10.1023/A:1025515504413.

Yan, Y., Li, Z.: 2006, Astrophys. J. 638, 1162. doi:10.1086/499064.

Yan, Y., Sakurai, T.: 1997, Solar Phys. 174, 65. doi:10.1023/A:1004943409775.

Yan, Y., Sakurai, T.: 2000, Solar Phys. 195, 89. doi:10.1023/A:1005248128673.

Yan, Y., Wang, J.: 1995, Astron. Astrophys. 298, 277.

Yan, Y., Deng, Y., Karlický, M., Fu, Q., Wang, S., Liu, Y.: 2001a, Astrophys. J. Lett. 551, L115. doi:10.1086/319829.

Yan, Y., Liu, Y., Akioka, M., Wei, F.: 2001b, Solar Phys. 201, 337. doi:10.1023/A:1017934615013.

Yan, Y., Pick, M., Wang, M., Krucker, S., Vourlidas, A.: 2006, Solar Phys. 239, 277. doi:10.1007/s11207-006-0202-6.

Yang, H.S., Zhang, H.M.: 1980, Acta Astron. Sinica 21, 136.

Yang, H.S., Hong, Q.F., Ding, Y.J.: 1988, Solar Phys. 117, 57. doi:10.1007/BF00148572.

Yang, W.H., Sturrock, P.A., Antiochos, S.K.: 1986, Astrophys. J. 309, 383. doi:10.1086/164610.

Yurchyshyn, V.B., Wang, H., Qiu, J., Goode, P.R., Abramenko, V.I.: 2000, Astrophys. J. 540, 1143. doi:10.1086/309359.

Zhang, Y., Wang, J., Attrill, G.D.R., Harra, L.K., Yang, Z., He, X.: 2007, Solar Phys. 241, 329. doi:10.1007/s11207-007-0229-3.

Zirin, H., Tanaka, K.: 1973, Solar Phys. 32, 173. doi:10.1007/BF00152736.

Zuccarello, F., Romano, P., Farnik, F., Karlicky, M., Contarino, L., Battiato, V., Guglielmino, S.L., Comparato, M., Ugarte-Urra, I.: 2009, Astron. Astrophys. 493, 629. doi:10.1051/0004-6361:200809887. 